Mathematical Modelling and Analysis

Volume 22 Number 2, March 2017, 157-166

https://doi.org/10.3846/13926292.2017.1277561

(c) Vilnius Gediminas Technical University, 2017
Publisher: Taylor\&Francis and VGTU

http://www.tandfonline.com/TMMA

ISSN: $1392-6292$

eISSN: 1648-3510

\title{
Two Area Preserving Maps from the Square to the $p$-Ball
}

\section{Adrian Holhoş ${ }^{a}$}

\author{
${ }^{a}$ Technical University of Cluj-Napoca \\ Memorandumului 28, 400114 Cluj-Napoca, Romania \\ E-mail(corresp.): adrian.holhos@math.utcluj.ro
}

Received July 13, 2016; revised December 21, 2016; published online March 1, 2017

\begin{abstract}
We construct two area preserving maps from the square to the $p$-ball (the domain defined by $|x|^{p}+|y|^{p} \leq|a|^{p}$ ) and use them to obtain low distorted uniform grids on the $p$-ball.
\end{abstract}

Keywords: area preserving map, distorsion, uniform grid, square, ball.

AMS Subject Classification: 65N50; 68U05; 65D18.

\section{Introduction}

In many applications in computer graphics, medical imaging, geosciences, one requires uniform and refinable grids on a given planar domain. In particular, one is interested in partitions having equal area cells with small diameter in order that no size distortions occur, but only small shape distortions. This goal can be achieved if we construct an area preserving map from the square to the given domain.

One of the most studied problem is how to map a square into a disc. There are many such maps (see [1], [5]) with different properties related to the distortions they introduce. One of these maps is the map introduced in [7], which is called the concentric map, because concentric squares are mapped to concentric circles. This map was reobtained in [2] in the study of partial differential equations on Lipschitz domains and also in [6] in the study of a new method to obtain uniform grids on the sphere. The concentric map is useful because it preserves the area, so a uniform distribution of points in the square will map to a uniform distribution of points in the disc. This fact was used in some sampling computations in computer graphics [4].

Our paper generalizes the concentric map. We obtain two area preserving maps from the square to the $p$-ball, i.e. two maps such that every subdomain of the square $[-r, r]^{2}$ is mapped into a subdomain of

$$
\mathcal{B}_{p}(a)=\left\{(x, y) \in \mathbb{R}^{2},|x|^{p}+|y|^{p} \leq a^{p}\right\}
$$


of the same area. These maps have the same good properties as the concentric map: equal-area preservation, bicontinuity (the map and its inverse are continuous) and low distortion.

The paper is structured as follows: in Section 2 we construct an area preserving map from $\mathbb{R}^{2}$ to $\mathbb{R}^{2}$ and its inverse, which will be used in Section 3 to obtain two maps from the square to the $p$-ball. Finally, in Section 4 we present some uniform and refinable grids on the $p$-ball obtained from uniform and refinable grids on the square.

\section{An area preserving map and its inverse}

For $p>0$ let $\mathcal{B}_{p}(a)$ be the $p$-ball of radius $a>0$, centered at the origin, defined by (1.1). The area of this $p$-ball can be computed using Beta and Gamma functions

$$
\begin{gathered}
\text { Area }\left(\mathcal{B}_{p}(a)\right)=4 \int_{0}^{a}\left(a^{p}-x^{p}\right)^{\frac{1}{p}} \mathrm{~d} x=4 a^{2} \frac{1}{p} \int_{0}^{1}(1-t)^{\frac{1}{p}} t^{\frac{1}{p}-1} \mathrm{~d} t \\
=4 a^{2} \frac{1}{p} \beta\left(\frac{1}{p}+1, \frac{1}{p}\right)=4 a^{2} \frac{\Gamma^{2}(1 / p+1)}{\Gamma(2 / p+1)}=2 c^{2} a^{2}
\end{gathered}
$$

with $c=\sqrt{2} \cdot \Gamma(1 / p+1) / \sqrt{\Gamma(2 / p+1)}$. We notice that for $p=1$ the value of $c$ is 1 and the radius of the ball $\mathcal{B}_{1}\left(a^{\prime}\right)$ must be $a^{\prime}=c a$ in order to have the same area as $\mathcal{B}_{p}(a)$.

Next, we construct the map $\mathcal{U}_{p}: \mathcal{B}_{p}(a) \rightarrow \mathcal{B}_{1}(c a)$ with the property that every subset of $\mathcal{B}_{p}(a)$ is mapped into a subset of $\mathcal{B}_{1}(c a)$ of the same area. For $(x, y) \in \mathcal{B}_{p}(a)$ let us denote $(X, Y)=\mathcal{U}_{p}(x, y)$.

Consider the bijection $F:[0,1] \rightarrow[0,1]$, a particular case of the regularized incomplete Beta function (also known in statistics as cumulative beta distribution)

$$
F(t)=\frac{1}{\int_{0}^{1}[u(1-u)]^{\frac{1}{p}-1} \mathrm{~d} u} \int_{0}^{t} u^{\frac{1}{p}-1}(1-u)^{\frac{1}{p}-1} \mathrm{~d} u=\frac{1}{p c^{2}} \int_{0}^{t}[u(1-u)]^{\frac{1}{p}-1} \mathrm{~d} u
$$

for $t \in[0,1]$. In the standard notation we have $F(t)=I_{t}(1 / p, 1 / p)$. One has $F(0)=0, F(1)=1$ and $F(1 / 2)=1 / 2$.

Theorem 1. The map $\mathcal{U}_{p}$ defined by

$$
\begin{aligned}
& X=\operatorname{sgn}(x) c \sqrt[p]{|x|^{p}+|y|^{p}} F\left(\frac{|x|^{p}}{|x|^{p}+|y|^{p}}\right), \\
& Y=\operatorname{sgn}(y) c \sqrt[p]{|x|^{p}+|y|^{p}}\left(1-F\left(\frac{|x|^{p}}{|x|^{p}+|y|^{p}}\right)\right) .
\end{aligned}
$$

has the area preserving property.

Proof. It is sufficient to consider the case $x, y \geq 0$. The area of the domain $\mathcal{B}_{p}(a)$ must be equal to the area of the domain $\mathcal{B}_{1}\left(a^{\prime}\right)$, so we obtain the relation 
$a^{\prime}=c a$. We will impose the condition that concentric $p$-circles are mapped into concentric 1-circles. The relation $a^{\prime}=c a$ implies $X+Y=c\left(x^{p}+y^{p}\right)^{1 / p}$.

Now, because $\mathcal{U}_{p}$ will be an area preserving map, its Jacobian is 1 . So, we have

$$
\frac{\partial X}{\partial x} \frac{\partial Y}{\partial y}-\frac{\partial X}{\partial y} \frac{\partial Y}{\partial x}=1
$$

Replacing $Y$ with $c\left(x^{p}+y^{p}\right)^{1 / p}-X$ we obtain the following first order partial differential equation:

$$
c y^{p-1} \frac{\partial X}{\partial x}-c x^{p-1} \frac{\partial X}{\partial y}=\left(x^{p}+y^{p}\right)^{1-\frac{1}{p}}
$$

We will impose also the condition $X(0, y)=0$. This means that the line $x=0$ is mapped into the line $X=0$. We have to solve the following symmetric system

$$
\frac{\mathrm{d} x}{c y^{p-1}}=\frac{\mathrm{d} y}{-c x^{p-1}}=\frac{\mathrm{d} X}{\left(x^{p}+y^{p}\right)^{1-1 / p}} .
$$

The first equation gives us $x^{p}+y^{p}=C_{1}$. Using this we obtain

$$
\frac{\mathrm{d} x}{c y^{p-1}}=\frac{\mathrm{d} X}{C_{1}^{1-1 / p}} \text { which is equivalent to } \frac{\mathrm{d} x}{\left(C_{1}-x^{p}\right)^{1-1 / p}}=c C_{1}^{1 / p-1} \mathrm{~d} X .
$$

By integration, we obtain

$$
\int \frac{\mathrm{d} x}{\left(C_{1}-x^{p}\right)^{1-1 / p}}=\int c C_{1}^{1 / p-1} \mathrm{~d} X
$$

Using the condition $X(0, y)=0$ and the relation (obtained by the change of variable $x^{p}=C_{1} u$ )

$$
\int_{0}^{x}\left(C_{1}-x^{p}\right)^{\frac{1}{p}-1} \mathrm{~d} x=\frac{1}{p} C_{1}^{\frac{2}{p}-1} \int_{0}^{\frac{x^{p}}{C_{1}}}(1-u)^{\frac{1}{p}-1} u^{\frac{1}{p}-1} \mathrm{~d} u=c^{2} C_{1}^{\frac{2}{p}-1} F\left(\frac{x^{p}}{C_{1}}\right)
$$

we get

$$
c^{2} C_{1}^{\frac{2}{p}-1} F\left(x^{p} / C_{1}\right)=c C_{1}^{\frac{1}{p}-1} X .
$$

Replacing $x^{p}+y^{p}=C_{1}$ in this last equality we obtain

$$
X=c \sqrt[p]{x^{p}+y^{p}} F\left(\frac{x^{p}}{x^{p}+y^{p}}\right)
$$

Finally, from the relation $X+Y=c\left(x^{p}+y^{p}\right)^{1 / p}$ we obtain the value of $Y$ given in (2.1).

Remark 1. Consider some particular cases for the map $\mathcal{U}_{p}$. In the case $p=1$, we have $F(t)=t$ and $c=1$. Thus $\mathcal{U}_{1}$ is the identity map $(X, Y)=(x, y)$. 
For the case $p=2$ we have $c=\frac{\sqrt{\pi}}{\sqrt{2}}$ and $F(t)=\frac{1}{\pi}\left(\arcsin (2 t-1)+\frac{\pi}{2}\right)$. The map $\mathcal{U}_{2}$ is given by

$$
\begin{aligned}
& X=\operatorname{sgn}(x) \frac{\sqrt{x^{2}+y^{2}}}{\sqrt{2 \pi}}\left(\frac{\pi}{2}+\arcsin \frac{x^{2}-y^{2}}{x^{2}+y^{2}}\right), \\
& Y=\operatorname{sgn}(y) \frac{\sqrt{x^{2}+y^{2}}}{\sqrt{2 \pi}}\left(\frac{\pi}{2}-\arcsin \frac{x^{2}-y^{2}}{x^{2}+y^{2}}\right) .
\end{aligned}
$$

In the limit case $p=\infty$, the ball $\mathcal{B}_{\infty}(a)$ represents the square $[-a, a]^{2}$. In this case $c=\sqrt{2}$ and

$$
\lim _{p \rightarrow \infty} F\left(\frac{|x|^{p}}{|x|^{p}+|y|^{p}}\right)= \begin{cases}1-\frac{|y|}{2|x|}, & |y|<|x|, \\ \frac{1}{2}, & |x|=|y|, \\ \frac{|x|}{2|y|}, & |x|<|y| .\end{cases}
$$

Indeed, consider the case $|x|<|y|$. We have

$$
\begin{aligned}
F\left(\frac{|x|^{p}}{|x|^{p}+|y|^{p}}\right) & =\frac{1}{c^{2} p} \int_{0}^{\frac{|x|^{p}}{|x|^{p}+|y|^{p}}}[u(1-u)]^{\frac{1}{p}-1} \mathrm{~d} u \\
& =\frac{1}{c^{2}} \int_{0}^{\frac{|x|}{\sqrt[p]{|x|^{p}+|y|^{p}}}}\left(1-t^{p}\right)^{\frac{1}{p}-1} \mathrm{~d} t .
\end{aligned}
$$

The inequalities $1 \leq\left(1-t^{p}\right)^{\frac{1}{p}-1} \leq\left(\frac{|y|^{p}}{|x|^{p}+|y|^{p}}\right)^{\frac{1}{p}-1}$ lead us to

$$
\frac{|x|}{c^{2} \sqrt[p]{|x|^{p}+|y|^{p}}} \leq F\left(\frac{|x|^{p}}{|x|^{p}+|y|^{p}}\right) \leq \frac{|x|}{c^{2} \sqrt[p]{|x|^{p}+|y|^{p}}} \cdot\left(\frac{|y|^{p}}{\left(|x|^{p}+|y|^{p}\right)}\right)^{\frac{1}{p}-1}
$$

and because $\lim _{p \rightarrow \infty} \sqrt[p]{|x|^{p}+|y|^{p}}=\max (|x|,|y|)=|y|$ we obtain

$$
\lim _{p \rightarrow \infty} F\left(\frac{|x|^{p}}{|x|^{p}+|y|^{p}}\right)=\frac{|x|}{2|y|}, \text { when }|x|<|y| \text {. }
$$

For the case $|y|<|x|$ we use the relation $F(t)=1-F(1-t)$ and we obtain

$$
\lim _{p \rightarrow \infty} F\left(\frac{|x|^{p}}{|x|^{p}+|y|^{p}}\right)=1-\frac{|y|}{2|x|}, \text { when }|y|<|x| \text {. }
$$

For the case when $|x|=|y|$ we use $F(1 / 2)=1 / 2$.

The map $\mathcal{U}_{\infty}$ is defined by

$$
\begin{aligned}
& X= \begin{cases}\sqrt{2} x-\frac{1}{\sqrt{2}} \operatorname{sgn}(x) \operatorname{sgn}(y) y, & |x| \geq|y|, \\
\frac{1}{\sqrt{2}} x, & |x|<|y|,\end{cases} \\
& Y= \begin{cases}\frac{1}{\sqrt{2}} y, & |x| \geq|y|, \\
\sqrt{2} y-\frac{1}{\sqrt{2}} \operatorname{sgn}(x) \operatorname{sgn}(y) x, & |x|<|y| .\end{cases}
\end{aligned}
$$

Let us also notice that the following relations hold true

$$
|X|+|Y|=\sqrt{2} \max (|x|,|y|) \text { and }|X|-|Y|=\sqrt{2}(|x|-|y|) .
$$


Theorem 2. The inverse of the map defined in Theorem 1 is given by

$$
\begin{aligned}
& x=\operatorname{sgn}(X) \frac{1}{c}(|X|+|Y|) \sqrt[p]{G(|X| /(|X|+|Y|))}, \\
& y=\operatorname{sgn}(Y) \frac{1}{c}(|X|+|Y|) \sqrt[p]{1-G(|X| /(|X|+|Y|))},
\end{aligned}
$$

where $G:[0,1] \rightarrow[0,1]$ is the inverse of the function $F$.

Proof. From the definition of the map $\mathcal{U}_{p}$ we have

$$
|X|+|Y|=c\left(|x|^{p}+|y|^{p}\right)^{\frac{1}{p}} \quad \text { and } \quad \frac{|X|}{|Y|}=\frac{F\left(\frac{|x|^{p}}{|x|^{p}+|y|^{p}}\right)}{1-F\left(\frac{|x|^{p}}{|x|^{p}+|y|^{p}}\right)} .
$$

We obtain

$$
F\left(\frac{|x|^{p}}{|x|^{p}+|y|^{p}}\right)=\frac{|X|}{|X|+|Y|}, \quad \text { so, we have } \frac{|x|^{p}}{|x|^{p}+|y|^{p}}=G\left(\frac{|X|}{|X|+|Y|}\right) .
$$

From this, because $\operatorname{sgn}(x)=\operatorname{sgn}(X)$, we finally get

$$
x=\operatorname{sgn}(X) \frac{|X|+|Y|}{c} \sqrt[p]{G\left(\frac{|X|}{|X|+|Y|}\right)} .
$$

Because $\frac{|y|^{p}}{|x|^{p}+|y|^{p}}=1-G\left(\frac{|X|}{|X|+|Y|}\right)$ we similarly obtain

$$
y=\operatorname{sgn}(Y) \frac{|X|+|Y|}{c} \sqrt[p]{1-G\left(\frac{|X|}{|X|+|Y|}\right)}
$$

Remark 2. For the map $\mathcal{U}_{p}^{-1}$ consider the same particular cases as for the map $\mathcal{U}_{p}$. In the case $p=1$ we have the identity map.

In the case $p=2$ the function $G$ is defined by $G(t)=\cos ^{2} \frac{\pi(1-t)}{2}$. The map $\mathcal{U}_{2}^{-1}$ is given by

$$
\begin{aligned}
& x=\operatorname{sgn}(X) \frac{\sqrt{2}}{\sqrt{\pi}}(|X|+|Y|) \cos \frac{\pi|Y|}{2(|X|+|Y|)} \\
& y=\operatorname{sgn}(Y) \frac{\sqrt{2}}{\sqrt{\pi}}(|X|+|Y|) \sin \frac{\pi|Y|}{2(|X|+|Y|)} .
\end{aligned}
$$

In the limit case $p=\infty$, the map $\mathcal{U}_{p}^{-1}$ can be obtained from $(2.2)$ and it is defined by

$$
\begin{aligned}
& x=\operatorname{sgn}(X) \frac{1}{\sqrt{2}}(|X|+\min (|X|,|Y|)), \\
& y=\operatorname{sgn}(Y) \frac{1}{\sqrt{2}}(|Y|+\min (|X|,|Y|)) .
\end{aligned}
$$




\section{Two maps from the square to the $p$-ball}

In this section we will obtain two maps from the square to the $p$-ball using the inverse of the map $\mathcal{U}_{p}$, which was defined in the previous section and we will study the distortions introduced by these maps. First, let us present how we will measure the distorsions of these two maps.

\subsection{Distorsions measurements}

We use the local isotropy distorsion [5], denoted $D_{I}$, which is the ratio of the semi-axes of an ellipse, called Tissot's indicatrix [8]. Tissot introduced this tool to measure the distorsions of any map from a sphere to a planar domain. For any point on the sphere an infinitesimal circle is mapped onto an infinitesimal ellipse on the plane. This ellipse, with semi-major axis $a$ and semi-minor axis $b$, describes the local characteristics of the map. For a map $(x, y) \longmapsto(U, V)$ from a planar domain to a planar domain, the formulae for $a$ and $b$ are

$$
\begin{aligned}
h & =\sqrt{\left(\frac{\partial U}{\partial x}\right)^{2}+\left(\frac{\partial V}{\partial x}\right)^{2}}, \quad k=\sqrt{\left(\frac{\partial U}{\partial y}\right)^{2}+\left(\frac{\partial V}{\partial y}\right)^{2}}, \\
s & =\frac{\partial U}{\partial x} \frac{\partial V}{\partial y}-\frac{\partial U}{\partial y} \frac{\partial V}{\partial x} \\
a & =\left(\sqrt{h^{2}+k^{2}+2 s}+\sqrt{h^{2}+k^{2}-2 s}\right) / 2, \\
b & =\left(\sqrt{h^{2}+k^{2}+2 s}-\sqrt{h^{2}+k^{2}-2 s}\right) / 2 .
\end{aligned}
$$

We have $s=a \cdot b$ and this is 1 for area preserving maps. The local isotropy distorsion $D_{I}$ is given by the relation

$$
D_{I}=\frac{a}{b}=\frac{h^{2}+k^{2}+\sqrt{\left(h^{2}+k^{2}+2 s\right)\left(h^{2}+k^{2}-2 s\right)}}{2 s} .
$$

When the map is area preserving the local isotropy distortion is given by

$$
D_{I}=\left(h^{2}+k^{2}+\sqrt{\left(h^{2}+k^{2}\right)^{2}-4}\right) / 2 .
$$

The smallest value of $D_{I}$, in this case, is 1 and this value is obtained when

$$
\frac{\partial U}{\partial x}=\frac{\partial V}{\partial y}, \quad \frac{\partial U}{\partial y}=-\frac{\partial V}{\partial x}
$$

i.e. the map is conformal. Let us remark that a map from a planar domain to a planar domain is both area preserving and conformal if and only if the map is isometric, i.e. it is length-preserving (a rotation, a translation, a reflection, or a composition of them).

\subsection{The first map from the square to the $p$-ball}

We define $\mathcal{T}_{p}:[-r, r]^{2} \rightarrow \mathcal{B}_{p}\left(\frac{\sqrt{2} r}{c}\right)$ by $\mathcal{T}_{p}=\mathcal{U}_{p}^{-1} \circ \mathcal{U}_{\infty}$. This map has the expression

$$
U=\operatorname{sgn}(x) \frac{\sqrt{2}}{c} \max (|x|,|y|) \sqrt[p]{G\left(\frac{\max (|x|,|y|)+|x|-|y|}{2 \max (|x|,|y|)}\right)}
$$




$$
V=\operatorname{sgn}(y) \frac{\sqrt{2}}{c} \max (|x|,|y|)\left(1-G\left(\frac{\max (|x|,|y|)+|x|-|y|}{2 \max (|x|,|y|)}\right)\right)^{1 / p} .
$$

In the particular case $p=2$ this map becomes

$$
\mathcal{T}_{2}: \quad(U, V)= \begin{cases}\left(\frac{2}{\sqrt{\pi}} x \cos \frac{y \pi}{4 x}, \frac{2}{\sqrt{\pi}} x \sin \frac{y \pi}{4 x}\right), & |x| \geq|y|>0, \\ \left(\frac{2}{\sqrt{\pi}} y \sin \frac{x \pi}{4 y}, \frac{2}{\sqrt{\pi}} y \cos \frac{x \pi}{4 y}\right), & |y|>|x|>0, \\ (0,0), & x=y=0,\end{cases}
$$

which is the concentric map, introduced in [7], also obtained in $[2,6]$.

The inverse $\mathcal{T}_{p}^{-1}$ is given by

$$
\begin{aligned}
& x=\operatorname{sgn}(U) \sqrt{2} c \sqrt[p]{|U|^{p}+||^{p}} \min \left(F\left(\frac{|U|^{p}}{|U|^{p}+|V|^{p}}\right), \frac{1}{2}\right), \\
& y=\operatorname{sgn}(V) \sqrt{2} c \sqrt[p]{|U|^{p}+|V|^{p}}\left[1-\max \left(F\left(\frac{|U|^{p}}{|U|^{p}+|V|^{p}}\right), \frac{1}{2}\right)\right] .
\end{aligned}
$$

In order to evaluate the distorsions introduced by the map $\mathcal{T}_{p}$ we have computed the value of $D_{I}$ using formula (3.1), where

$$
\begin{aligned}
h^{2}+k^{2}= & \frac{c^{2}}{2}\left(1+t^{2}\right)\left[g^{2-\frac{2}{p}}+(1-g)^{2-\frac{2}{p}}\right]+\frac{2}{c^{2}}\left[g^{\frac{2}{p}}+(1-g)^{\frac{2}{p}}\right] \\
& +2 t\left[g^{1-\frac{1}{p}}(1-g)^{\frac{1}{p}}-g^{\frac{1}{p}}(1-g)^{1-\frac{1}{p}}\right]
\end{aligned}
$$

with $g=G(t / 2)$ and $t=\min (|x| /|y|,|y| /|x|)$.
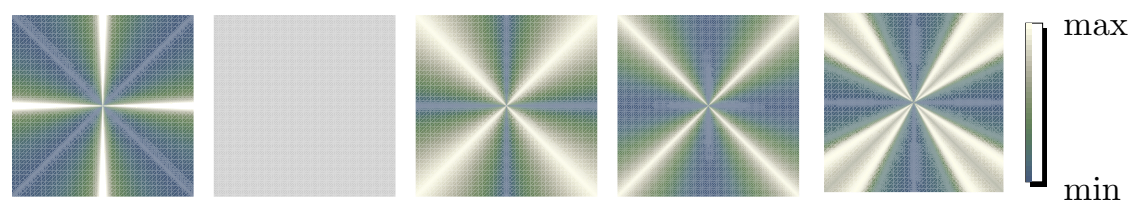

Figure 1. The value of $D_{I}$ for the map $\mathcal{T}_{p}$ for $p=\frac{2}{3}, 1, \frac{3}{2}, 2,10$.

Figure 1 shows the value of $D_{I}$ for the map $\mathcal{T}_{p}$ for some values of $p$. For $p \in(0,1)$ the value of $D_{I}$ grows indefinitely on the lines $x=0$ and $y=0$. For $p=1$ the value of $D_{I}$ is constant $(3+\sqrt{5}) / 2$. For $p>1$ the maximum value of $D_{I}$ is finite and depends on $p$. In particular, for $p=2$ the value of $D_{I}$ is given by

$$
D_{I}=\frac{\pi}{8}\left(1+t^{2}\right)+\frac{2}{\pi}+\sqrt{\left(\frac{\pi}{8}\left(1+t^{2}\right)+\frac{2}{\pi}\right)^{2}-1}, \quad t=\min \left(\frac{|x|}{|y|}, \frac{|y|}{|x|}\right) .
$$

\subsection{The second map from the square to the $p$-ball}

The map we have constructed is not unique, there are many other area preserving maps from the square to the $p$-ball. For example, a different such area 
preserving map can be constructed as follows: we start with the map from the square $[-r, r]^{2}$ onto the rhombus $|X|+|Y| \leq \sqrt{2} r$ given by

$$
\mathcal{S}: \quad(X, Y)=\frac{1}{\sqrt{2}}(x-y, x+y),
$$

which is actually a rotation with $\pi / 4$ in the trigonometric sense, and we compose it with the inverse of $\mathcal{U}_{p}$. Thus, we obtain the map $\mathcal{S}_{p}=\mathcal{U}_{p}^{-1} \circ \mathcal{S}$, given by

$$
\begin{aligned}
& U=\frac{1}{c \sqrt{2}} \operatorname{sgn}(x-y)(|x-y|+|x+y|)\left(G\left(\frac{|x-y|}{|x-y|+|x+y|}\right)\right)^{1 / p}, \\
& V=\frac{1}{c \sqrt{2}} \operatorname{sgn}(x+y)(|x-y|+|x+y|)\left(1-G\left(\frac{|x-y|}{|x-y|+|x+y|}\right)\right)^{1 / p} .
\end{aligned}
$$

Through this map the square $[-r, r]^{2}$ is mapped into the $p$-ball $\mathcal{B}_{p}\left(\frac{\sqrt{2} r}{c}\right)$. The map $\mathcal{S}_{p}$ has the property of area preserving, being the composition of two area preserving maps. The inverse $\mathcal{S}_{p}^{-1}$ is given by

$$
\begin{aligned}
& x=\frac{c \sqrt[p]{|U|^{p}+|V|^{p}}}{\sqrt{2}}\left[\operatorname{sgn}(U) F\left(\frac{|U|^{p}}{|U|^{p}+|V|^{p}}\right)+\operatorname{sgn}(V)\left[1-F\left(\frac{|U|^{p}}{|U|^{p}+|V|^{p}}\right)\right]\right], \\
& y=\frac{c \sqrt[p]{|U|^{p}+|V|^{p}}}{\sqrt{2}}\left[\operatorname{sgn}(V)\left[1-F\left(\frac{|U|^{p}}{|U|^{p}+|V|^{p}}\right)\right]-\operatorname{sgn}(U) F\left(\frac{|U|^{p}}{|U|^{p}+|V|^{p}}\right)\right] .
\end{aligned}
$$

For the analysis of distorsions we use (3.1), with the same formula (3.2) for computing $h^{2}+k^{2}$, but with $g=G\left(\frac{1+t}{2}\right)$ and $t=-x / y$, if $|y| \geq|x|$, or $t=-y / x$, if $|y|<|x|$.
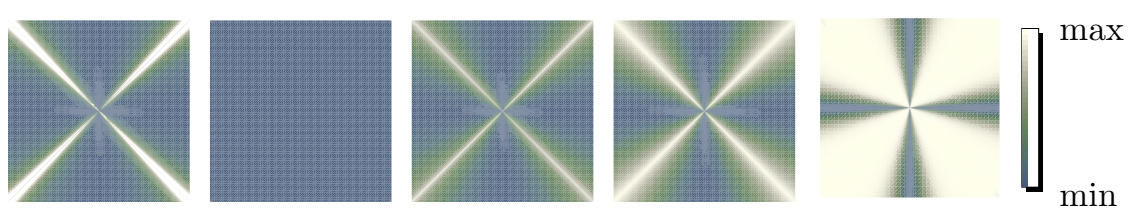

Figure 2. The value of $D_{I}$ for the map $\mathcal{S}_{p}$ for $p=\frac{2}{3}, 1, \frac{3}{2}, 2,10$.

In Figure 2 we have represented the value of $D_{I}$ for the map $\mathcal{S}_{p}$ for some values of $p$. For $p \in(0,1)$ the value of $D_{I}$ grows indefinitely on the lines $y= \pm x$. For $p=1$, the value of $D_{I}$ is 1 everywhere. This is true, because, in this case, the map is just a rotation. For $p>1$ the maximum value of $D_{I}$ is finite and depends on $p$. For $p=2$ the distorsion is given by the same formula (3.3).

\section{Uniform and refinable grids on the $p$-ball}

We can use the two area preserving maps, $\mathcal{T}_{p}$ and $\mathcal{S}_{p}$, to construct uniform and refinable grids on the $p$-ball starting from uniform and refinable grids on the square. 

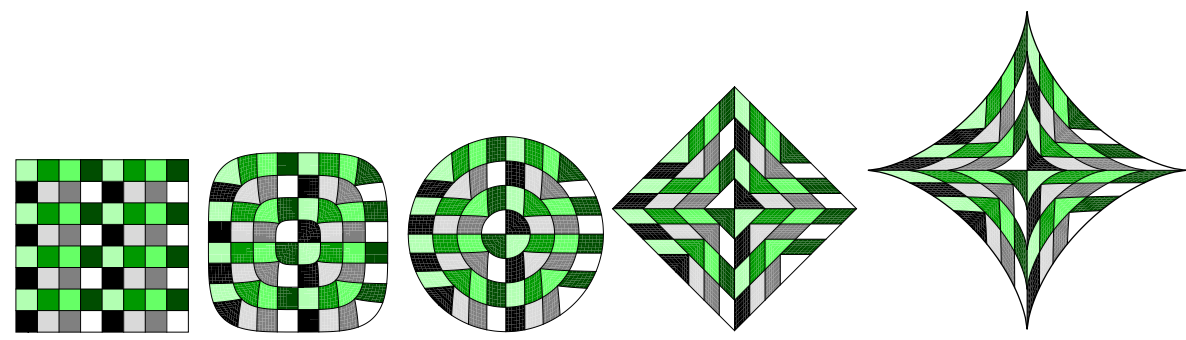

Figure 3. The image of the square from the left through the map $\mathcal{T}_{p}$ for $p=4,2,1,2 / 3$.

First, let us consider the regular square grid. In Figure 3 we have represented the image of the square from left through the map $\mathcal{T}_{p}$ for some values of $p$. In the case $p=4$, we obtain a uniform grid on the squircle (we use the notion of "squircle", not in the sense of Fernández Guasti [3], but as a special case of a superellipse). For $p=2$, we have a grid on the circle, for $p=1$, a grid on the diamond(rhombus) and for $p=\frac{2}{3}$, a uniform and refinable grid on the astroid. We can remark that the shape of the little squared cells is better preserved in the cases $p=2$ and $p=4$ for those cells situated on the vertical and horizontal lines passing through the center of the ball. We can also remark the greatest distorsion of shape, in the case of the cells situated on the diagonals $y= \pm x$.
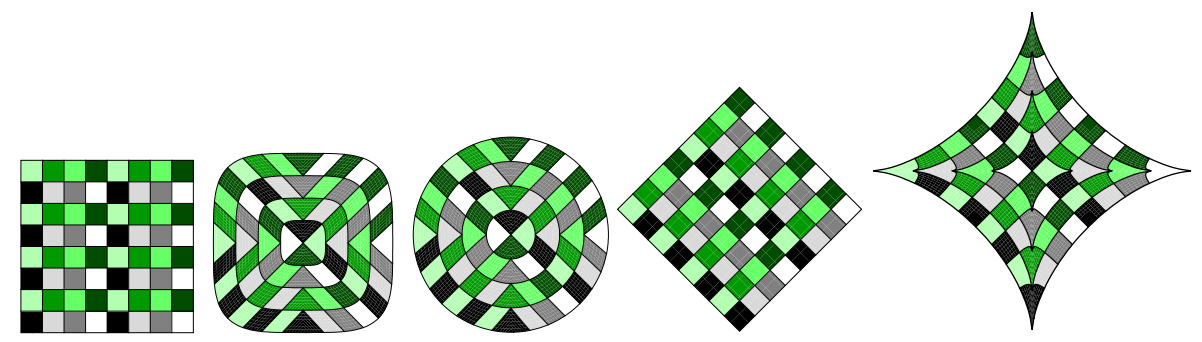

Figure 4. The image of the square from the left through the map $\mathcal{S}_{p}$ for $p=4,2,1,2 / 3$.

In Figure 4 we have represented the image of the square from the left through the map $\mathcal{S}_{p}$, for the same cases: squircle $(p=4)$, circle $(p=2)$, diamond(rhombus) $(p=1)$ and astroid $\left(p=\frac{2}{3}\right)$. We can remark the smallest distorsion on the lines $y= \pm x$ and the greatest distorsion on the vertical and horizontal lines passing through the center of the balls. We can also notice that the little "squared" cells are rotated anticlockwise with an angle of $\pi / 4$ from the original ones.

Now, by overlapping the uniform grids obtained through the maps $\mathcal{T}_{p}$ and $\mathcal{S}_{p}$ we get new grids on the $p$-ball. These new grids have triangular shaped cells. In Figure 5 we have represented these grids and it can be seen that the "triangular" cells look almost the same. Visually, the differences of shape between the cells are smaller than in the case of the square grids. Let us remark one more thing about these triangular grids: they can be obtained also, using only one of the maps we have introduced, either $\mathcal{T}_{p}$ or $\mathcal{S}_{p}$, by mapping the 
triangular grid of the square from the left.
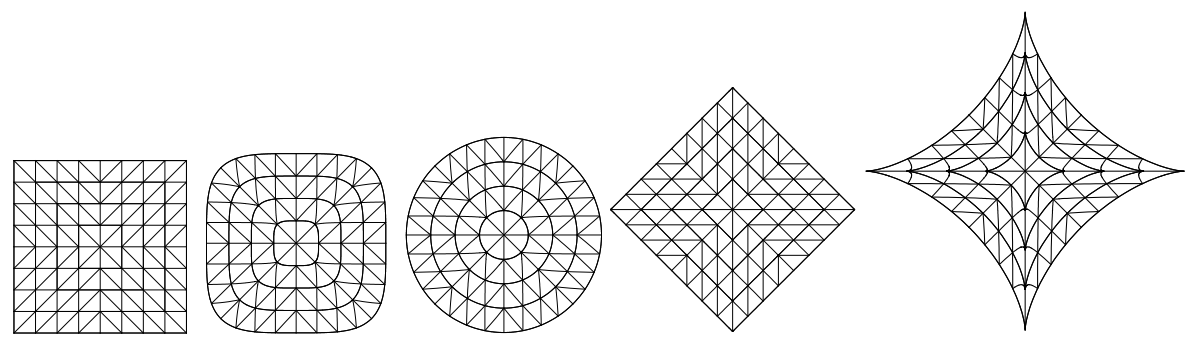

Figure 5. The images of the uniform grid of the square from left through the maps $\mathcal{T}_{p}$ or $\mathcal{S}_{p}$ for $p=4,2,1$ and $2 / 3$.

\section{References}

[1] C. Fong. Analytical methods for squaring the disc. 2014. http://arxiv.org/abs/1509.06344

[2] J.A. Griepentrog, W. Höppner, H.-C. Kaiser and J. Rehberg. A bi-Lipschitz continuous, volume preserving map from the unit ball onto a cube. Note di Matematica, 1:177-193, 2008. https://doi.org/10.1285/i15900932v28n1p177.

[3] M. Fernandez Guasti. Analytic geometry of some rectilinear figures. Int. J. Math. Educ. Sci. Technol., 23(6):895-901, 1992.

[4] C. Kolb, D. Mitchell and P. Hanrahan. A realistic camera model for computer graphics. In S. G. Mair and R. Cook(Eds.), Proceedings of the 22nd annual conference on Computer graphics and interactive techniques, SIGGRAPH '95, pp. 317-324. ACM, New York, 1995.

[5] M. Lambers. Mappings between sphere, disc, and square. Journal of Computer Graphics Techniques (JCGT), 5(2):1-21, 2016.

[6] D. Roşca. New uniform grids on the sphere. Astronomy \& Astrophysics, 520(A63), 2010. https://doi.org/10.1051/0004-6361/201015278.

[7] P. Shirley and K. Chiu. A low distortion map between disk and square. Journal of Graphics Tools, 2(3):45-52, 1997. https://doi.org/10.1080/10867651.1997.10487479.

[8] J. Snyder. Map projections-a working manual. U.S. Geological survey Professional Paper 1395. U.S. Government Printing Office, Washington, 1987. 